\title{
Beat-to-beat Variability of Microvascular Peripheral Resistances Assessed with a Non-invasive Approach
}

\author{
A Porta $^{1}$, G Baselli ${ }^{1}$, L Mainardi $^{2}$, D Lucini $^{3}$, M Pagani $^{3}$, A Malliani $^{3}$, S Cerutti $^{2}$ \\ 1Dipartimento di Elettronica per l'Automazione, Universita' di Brescia, Italia \\ 2Dipartimento di Bioingegneria, Politecnico di Milano, Milano, Italia \\ ${ }^{3}$ Medicina II, Ospedale L. Sacco, Universita' di Milano, Milano, Italia
}

\begin{abstract}
The pressure-flow relationship at peripheral level is non-invasively studied in human subjects: the impedance function and the beat-to-beat variability series of microvascular peripheral resistance are estimated. The frequency content of this variability signal is compared to those of more classical variability series at rest and during mild supine physical exercise.
\end{abstract}

\section{Introduction}

While the origin of the high frequency (HF) fluctuations (synchronous with respiratory activity) on the arterial pressure (AP) has been early clarified [1], several different mechanisms have been evoked to explain the low frequency (LF) rhythms (around $0.1 \mathrm{~Hz}$ ) [1]. Endogenous rhythmicities related to the activity of central oscillators, oscillations of feedback control circuits (i.e. baroceptor, chemoceptor and brain perfusion control system), autorhythmicities of the vascular resistances can contribute to the LF oscillations of AP. It has been proved that even isolated and denervated small blood vessels exhibit rhythmic contractions (i.e. vasomotion) [2]. To determine an effect at systemic level several peripheral vascular districts have to be tuned and the synchronisation is likely to be carried out by sympathetic control mechanisms.

In order to evaluate the contribution of changes in the microvascular resistance to the LF oscillations observed in AP and heart period (HP) variabilities, the blood flow (BF) was non-invasively recorded from the palmar region of human subjects by means of Laser-Doppler technique.

A sympathetic activation produced by a mild supine cycloergometer exercise was used to verify whether the sympathetic stimulation can be a possible mechanism able to alter the time course of the peripheral resistance and the coupling between the systemic and peripheral districts.

\section{Experimental protocol}

ECG, AP (via FINAPRES), respiratory movements (via thoracic belt), and skin BF (via Laser-Doppler technique) were recorded in 13 normal subjects ( 6 men and 7 women). The skin BF was recorded from the palmar region on the same hand utilised for the finger pressure measurement. The vascular impedance and resistance must be evaluated from simultaneous measures of AP and BF carried out at the same site of interest. Therefore the measures of $\mathrm{AP}$ and $\mathrm{BF}$ were performed as close as possible.

The Laser-Doppler flowmeter is based on the Doppler shift of the reflected light due to the relative movement of the blood. Thus it permits to obtain a rough measure of the mean blood flow velocity in the considered region. To determine a flow measure expressed in $\mathrm{cm}^{3} / \mathrm{sec}$ it would be necessary to measure the average vascular diameter and other geometrical parameters. Since these are not available, the skin BF is calibrated in Laser-Doppler shift units (LDsu) which are proportional to flow under the hypothesis that unknown parameters are kept constant within the experiment.

All the signals were recorded at rest (15 minutes) and during a mild (10\% of the nominal maximum) supine cycloergometer exercise (15 minutes). The recordings were sampled at $300 \mathrm{~Hz}$ and processed on a 486 PC.

\section{Methods}

\subsection{Estimation of the vascular impedance}

The Fourier Transform (FT) is used to estimate the transfer function between $\mathrm{AP}$ and $\mathrm{BF}$.

Let us indicate with 
$P_{k}(\omega)=\sum_{t=0}^{N-1} p_{k}(t) e^{-j \omega t} \quad$ and $\quad F_{k}(\omega)=\sum_{t=0}^{N-1} f_{k}(t) e^{-j \omega t}$

the FTs of the AP and BF signals $\left(p_{k}(t)\right.$ and $f_{k}(t)$ respectively) in the $\mathrm{k}$-th frame, where $\mathrm{t}$ and $\omega$ are the current time and angular frequency and $\mathrm{N}$ is the frame length. In the FT technique the frequency resolution increases with the frame length, conversely the variance of the estimate. To improve the consistency of the estimate the cross-spectrum $\mathrm{C}_{\mathrm{pr}}(\omega)$ between the AP and BF signals was calculated as

$\mathrm{C}_{\mathrm{pf}}(\omega)=\frac{1}{\mathrm{NK}_{\mathrm{k}=1}} \sum_{\mathrm{k}}^{\mathrm{K}} \mathrm{P}_{\mathrm{k}}(\omega) \mathrm{F}_{\mathrm{k}}^{*}(\omega)$,

while the spectrum $\mathrm{S}_{\mathrm{f}}(\omega)$ of $\mathrm{BF}$ signal was obtained as

$\mathrm{S}_{\mathrm{f}}(\omega)=\frac{1}{\mathrm{NK}} \sum_{\mathrm{k}=1}^{\mathrm{K}} \mathrm{F}_{\mathrm{k}}(\omega) \mathrm{F}_{\mathrm{k}}^{*}(\omega)$,

where $K$ is the total frame number. A frame length $N$ of 4096 samples was chosen, yielding a frequency resolution of $0.073 \mathrm{~Hz}$. Nine successive frames $(K=9)$, cosine windowed and $50 \%$ overlapped were taken to estimate the vascular impedance $Z(\omega)$ as

$Z(\omega)=\frac{C_{p f}(\omega)}{S_{f}(\omega)}$.

The mean resistance $R=Z(0)$ (i.e. the impedance at zero frequency) was evaluated directly as the ratio between the mean AP and the mean BF over the entire time window (20480 samples, i.e. $68.27 \mathrm{sec})$.

\subsection{Beat-to-beat series of peripheral resistances}

All the signals were processed in order to extract the beat-to-beat series able to evidence the effect of the control of the cardiovascular function performed by the autonomic nervous system. The beat-to-beat series were extracted according to the conventions shown in Fig.1. The measure of the cardiac cycle duration was taken as the time distance between two consecutive R-peaks (t(i), $\mathrm{i}$ is the cardiac cycle number). The systolic (s(i)) value was detected in the i-th cardiac cycle. The $i$-th diastolic (d(i)) value followed the $i$-th systolic value. The respiratory signal was sampled in correspondence to the first R-peak (resp(i)) in the $\mathrm{i}$-th cardiac cycle. The peripheral resistance value (r(i)) was obtained as the ratio between the mean AP and the mean BF in the $i$-th cardiac cycle as $r(i)=\frac{\frac{1}{t(i)} \int p(t) d t}{\frac{1}{t(i)} \int f(t) d t}$.

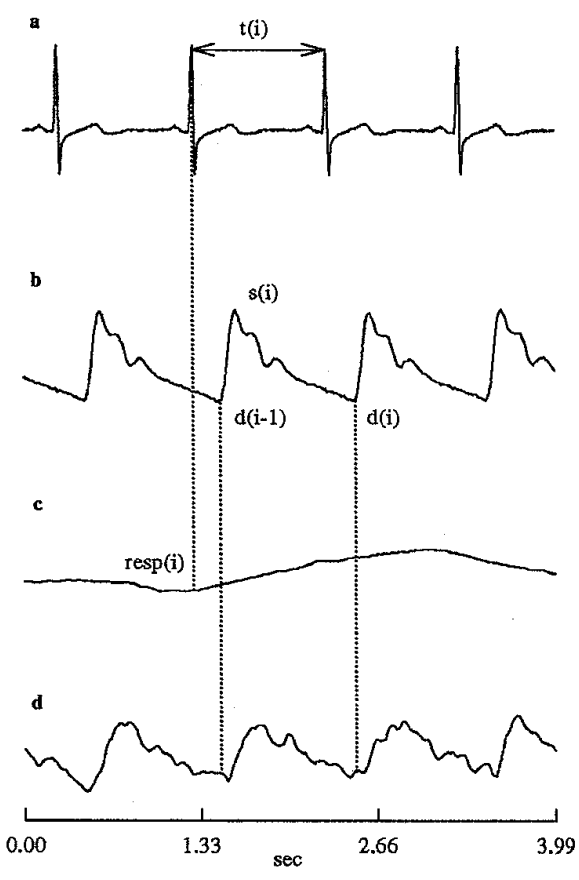

Figure 1. Conventions for the measure of $t(i), s(i), d(i)$, resp(i) and r(i) from ECG (a), AP (b), respiration (c) and skin $B F(d)$ signals.

All the beat-to-beat variability series were analysed by means of the autoregressive power spectral method. This method permits to relate each rhythm detected in the variability series to a real pole or a pair of complex and conjugated poles inside the unit circle of the complex zplane. Therefore pictures representing the position of the detected poles in the unit circle can be drawn.

\section{Results}

The power spectral densities of the AP and BF signals shown in Fig.2a,b evidence that, in addition to the cardiac-related rhythmicities, slow oscillations are present in both signals. Since almost all the power is localised around the multiples of the cardiac frequency, the coherence function between the AP and BF signals (Fig.2d) is high only in correspondence to these components. Therefore the estimated impedance function (Fig.2c, dashed line) was interpolated in correspondence to the points characterised by an high coherence value. This permits to obtain a more readable representation of the impedance function (Fig2c, solid line). It evidences a clear minimum around $1 \mathrm{~Hz}$ (i.e. around the cardiac frequency). The values of the impedance over $1 \mathrm{~Hz}$ are 

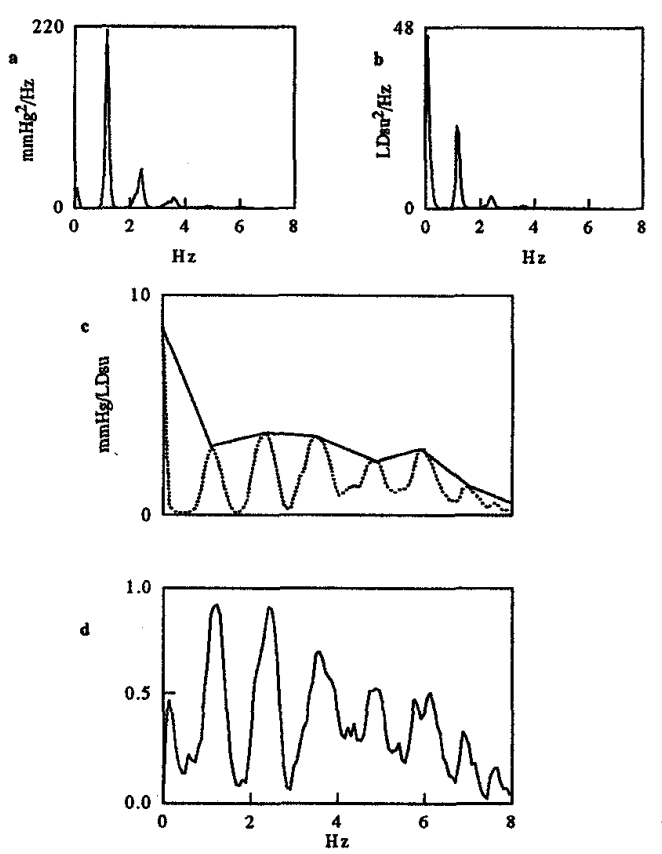

Figure 2. Periodograms of AP (a) and BF (b) signals. Modulus of the impedance function (c, dashed line) and its linear interpolation (c, solid line) between points with high coherence values. Coherence function (d).

almost constant and could be related to the characteristic impedance of the microvascular system.

The impedance analysis provides the average features of the pressure-flow relationship in a great detail. Nonetheless, its parameters are continuously varied from beat to beat according to the cardiovascular variability. Unfortunately an impedance analysis is difficult to be carried out on a beat-to-beat basis and, therefore, only the variability of the resistance value (i.e. the mean AP over the mean BF in a beat) is considered. The microvascular resistance values show rhythmic patterns even though sparse bursts related to a strong reduction of the $\mathrm{BF}$ can be found. As shown in Fig.3 the variability series of the peripheral resistance exhibits oscillations slower than those observed in the HP and AP variabilities. This suggests a way to address the variations in the center frequency of $L F$, as observed in different signals. The microvascular activity displays a rhythm in the LF region usually centered between 0.035 and $0.075 \mathrm{~Hz}$ (henceforth indicated as $\mu \mathrm{LF}$ ), while the frequency of the $\mathrm{LF}$ oscillation from HP and SAP variability lies usually between 0.075 and $0.15 \mathrm{~Hz}$. The $\mathrm{HF}$ rhythms is synchronous with respiration in all variability signals. Different symbols were chosen to represent poles in
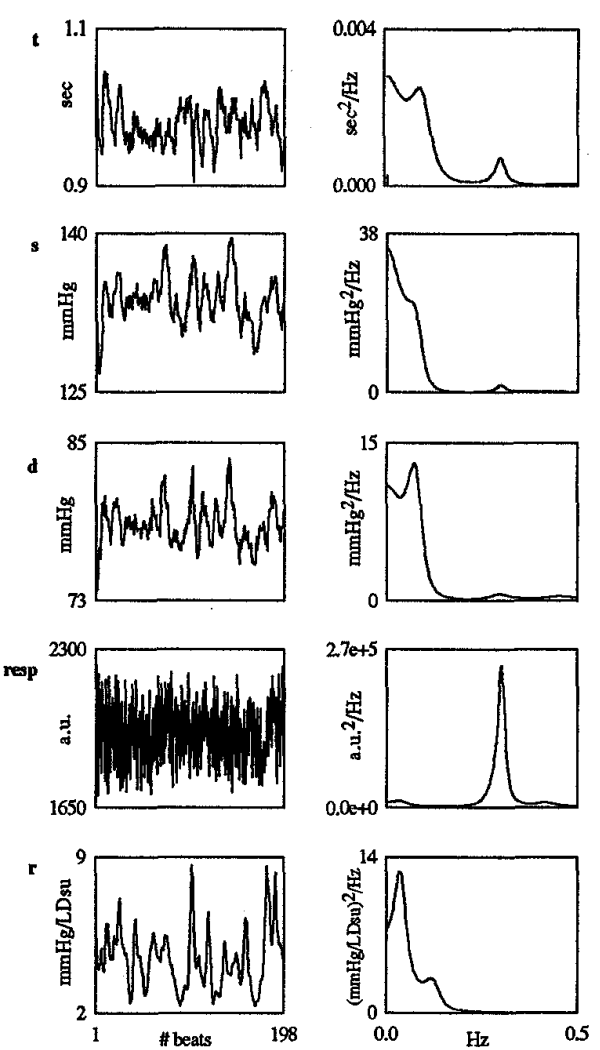

Figure 3. Variability series of $t(i), d(i), s(i)$, resp(i) and rii) values and relevant power spectral densities at rest

different frequency regions: triangle for the $\mu \mathrm{LF}$, circle for the LF, square for the HF.

The distribution of the poles at rest (Fig 4) evidences that $\mu \mathrm{LF}$ rhythmicities are frequently found (8/13) in the variability series of the resistance value. The frequency of these oscillations is $0.048 \pm 0.005 \mathrm{~Hz}$ (meantste). This rhythm is not detected $(1 / 13)$ in the HP variability ( $L F=0.1 \pm 0.004, H F=0.26 \pm 0.017$ ), while it appears in 4 and 5 subjects in the systolic and diastolic variabilities respectively in addition to the usual components $(\mathrm{LF}=0.1 \pm 0.005, \mathrm{HF}=0.27 \pm 0.017$ ). During exercise an increase of the mean resistance $(174 \pm 73 \%)$ is observed with a negligible change in the variance. Poles of $\mu \mathrm{LF}$ type are still present (9/13) in the variability series of the peripheral resistance; they are more clearly separated from LF poles. In addition poles (Fig.5) with a frequency of $0.058 \pm 0.002 \mathrm{~Hz}$ are found more frequently $(8 / 13)$ in the diastolic variability than in the systolic one $(3 / 13)$. By comparing Fig. 4 with Fig.5 it can be noted that the poles detected in all the variability signals are better clustered 


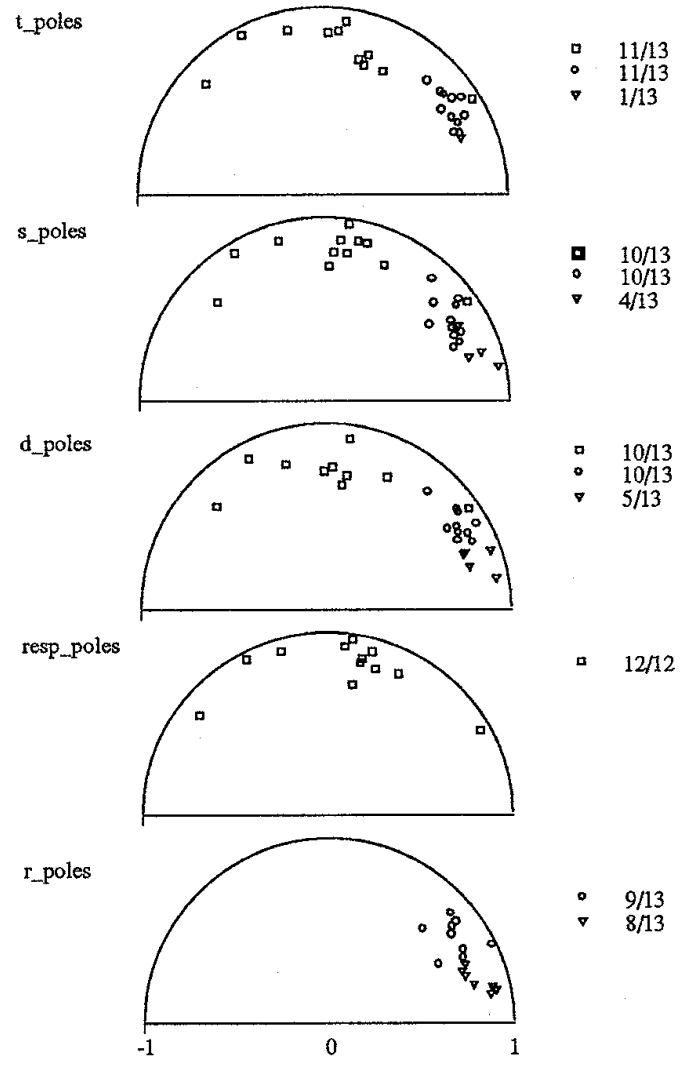

Figure 4. Pole distributions at rest.

during the sympathetic stimulation obtained by physical exercise than at rest.

\section{Conclusions}

The beat-to-beat variability of the resistance value exhibits rhythmic patterns. The frequency content of this vasomotor activity is shifted toward lower frequencies (around $0.05 \mathrm{~Hz}$ ) compared to those of the HP and AP variability series. These vasomotor waves are not evident in $\mathrm{HP}$ variability and seldom observed in the AP variabilities. The mild sympathetic activation produced by physical exercise determines an increase of the mean resistance value and appears to be able to synchronise vasomotor activity and its systemic effects detected on diastolic AP variability. The stronger tuning of the respiratory-related thythms could be due to the regularity of respiration during exercise.

The analysis of the pressure-flow relationship at the level of the microvascular district could permit a better understanding of the origin of the LF oscillations: changes in the frequency of the $L F$ rhythms could be

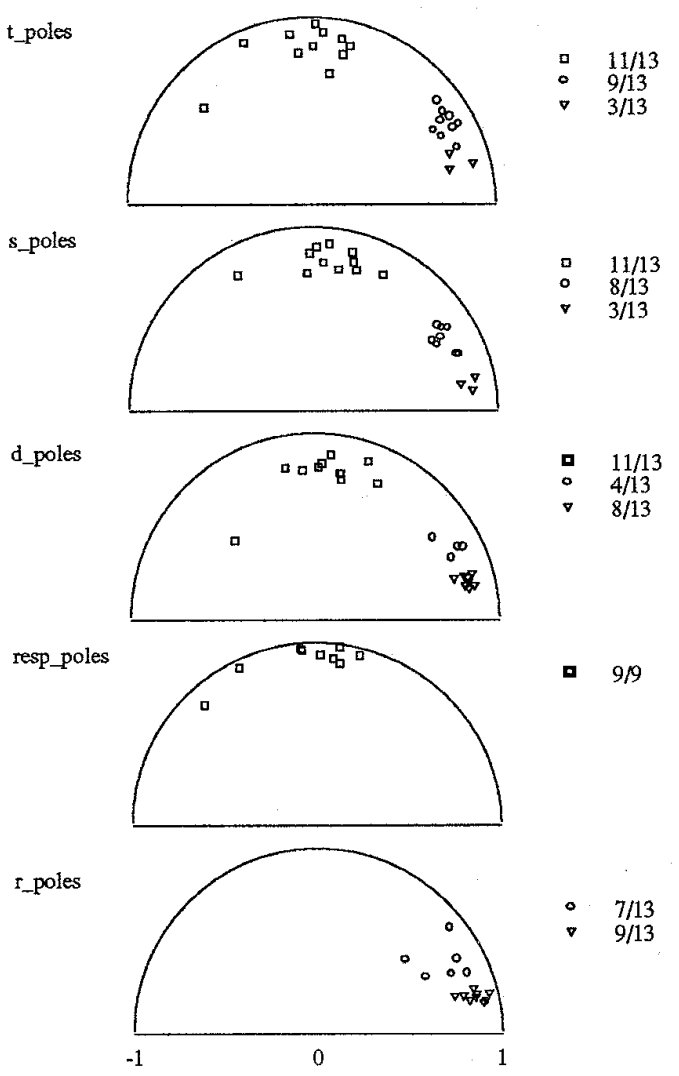

Figure 5. Pole distributions during exercise.

explained by means of several interacting oscillatory mechanisms [3] whose importance and coupling are varied by the autonomic nervous system. Moreover this analysis can extract in a non-invasive way, some clinical parameters useful to characterise and detect pathological conditions related to the microvascular system.

\section{References}

[1] Koepchen HP, History of Studies and Concepts of Blood Pressure Waves. in Mechanisms of Blood Pressure Waves. Miyakawa K, et al. eds., Springer Verlag, pp.3-23, 1984.

[2] Messmer K. and Hammersen F., Vasomotion and Quantitative capillaroscopy, S. Karger, 1983.

[3] Baselli G, Porta A, Ferrari G, Cerutti A, Pagani M, Rimoldi O, Multi-variate Identification and Spectral Decomposition for the Assessment of Cardiovascular Control, in Computer Analysis of Cardiovascular Signals, M. Di Rienzo et al. eds. IOS Press, Amsterdam, pp. 95$103,1995$.

Address for correspondence

Dr Alberto Porta, Universita' di Brescia, Dip. di Elettronica per l'Automazione, via Branze 38, 25123 Brescia, Italia 